\title{
Brugada Type 1 ECG Caused by an RVOT Tumor
}

\section{Bruno Schnegg, Jens Seiler, Thomas M Suter, Hildegard Tanner and Helge Servatius*}

\author{
Department of Cardiology, Inselspital, Bern University Hospital, University of Bern, Switzerland
}

\begin{abstract}
A 66-year-old female patient without any relevant medical history other than rectal cancer was admitted for biopsy of a peri-myocardial tumor after the incidental finding of a mass in the region of the ventral right ventricle in a followup positron emission tomography with computed tomography (PET-CT). Diagnostic investigation revealed a cardiac metastasis of the rectal cancer affecting the anterior right ventricle, obstructing the right ventricular outflow tract, and causing a type 1 Brugada ECG.
\end{abstract}

Keywords

Arrhythmia, Tumor, Right ventricle, Brugada ECG

\section{Introduction}

Obvious cardiac metastases are rare. The reported incidence in autopsies of patients with malignant neoplasms ranges from $2 \%$ to $20 \%$. Colon carcinoma is responsible for $1.2 \%$ of all secondary cardiac tumors [1]. Clinical presentations of cardiac metastases are variable and depend on the localization of the tumor. In intramyocardial tumors, arrhythmia is the leading presentation. With progression of infiltration, diastolic and systolic dysfunction may develop. ECG changes in patients with cardiac metastases are unspecific and usually show signs of pericardial effusion or myocardial ischemia [2]. ECG changes however, particularly changes in the ST segment and T wave, are highly suggestive of cardiac involvement in patients with neoplasia if reversible causes, as ischemia and peri-myocarditis are excluded [3].

\section{Case Presentation}

A 66 year-old female without any relevant medical history other than a rectal carcinoma (T3NOM0) that was surgically completely removed 6 months earlier, was admitted for a computed tomography (CT) guided biopsy (Figure 1) of a mass located in the peri-myocardial region of the right ventricular outflow tract (RVOT). A previously positron emission tomography performed for follow-up of colonic surgery confirmed a metabolically active tumor. Based on imaging, it was not possible to determine whether the mass was located in the pericardium or myocardium.

At the current hospital admission, the patient was free of any symptoms. Particularly, the patient's history was negative for syncope, palpitations and chest pain. The family history was negative for sudden cardiac death, arrhythmia or stillbirth. The physical examination was unrevealing.

The ECG at admission showed an elevated J-point of $0.25 \mathrm{mV}$ $(2.5 \mathrm{~mm})$, a coved ST-segment elevation and a negative $\mathrm{T}$ wave in lead V1, and T-wave inversions in V2 to V4. The Corrado index [3] was 1.2 in V1, consistent with type 1 Brugada ECG [4] (Figure 2). An ECG performed six months earlier, prior to diagnosis of the tumor in the right ventricle, showed an rSr' pattern and neither ST element elevation in $\mathrm{V} 1$ nor unequivocally negative $\mathrm{T}$ waves in $\mathrm{V} 1$ to V4 (Figure 3).

A needle biopsy of the mass was performed, and the histopathologic examination was consistent with partially necrotized tissue. Immunohistochemistry was positive for cytokeratin and p63, consistent with a metastasis of the rectal carcinoma. Immediately after the cardiac biopsy, a hemodynamic unstable wide complex tachycardia, most likely ventricular tachycardia, occurred necessitating electrical cardioversion.

The follow-up PET-CT also showed several new hyperdense lesions in the lungs, consistent with pulmonary metastases. Removal of the peri-myocardial mass was considered to be of an unfavorable risk-benefit ratio, and a palliative treatment strategy was chosen in view of the poor prognosis. The Type 1 Brugada ECG pattern persisted during follow-up ECG recordings.

\section{Discussion}

The case presented here demonstrates a newly developed Brugada type 1 ECG in a patient with a cardiac metastasis of a rectal carcinoma.

The ECG performed 6 months prior to the diagnosis of the right ventricular metastasis showed an rSr' configuration in $\mathrm{V} 1$ without meeting the criteria for complete right bundle block [5] or any criteria for a Brugada pattern. According to a current consensus report for

*Corresponding author: Helge Servatius, Department of Cardiology, Inselspital, Bern University Hospital, University of Bern, Freiburgstrasse, Bern, Switzerland, E-mail: helge.servatius@insel.ch

Received: August 07, 2016: Accepted: September 08, 2016: Published online: September 10, 2016

Citation: Schnegg B, Seiler J, Suter TM, et al. (2016) Brugada Type 1 ECG Caused by an RVOT Tumor. Scientific Pages Heart 1:002.

Copyright: (c) 2016 Schnegg B, et al. This is an open-access article distributed under the terms of the Creative Commons Attribution License, which permits unrestricted use, distribution, and reproduction in any medium, provided the original author and source are credited. 


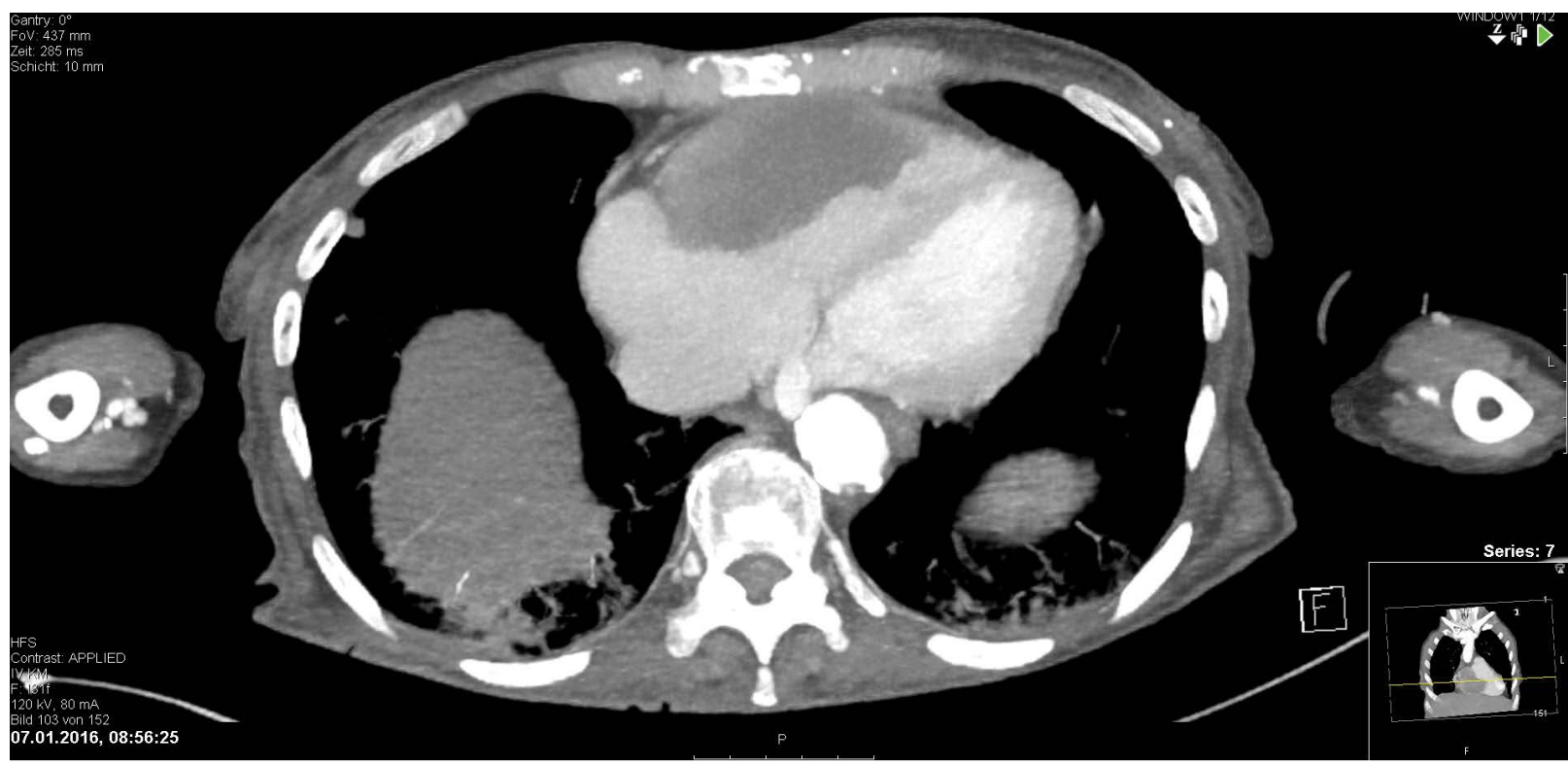

Figure 1: Computed tomography scan of the chest. The asterisk marks the tumor mass. RV = right ventricle, and LV = left ventricle.

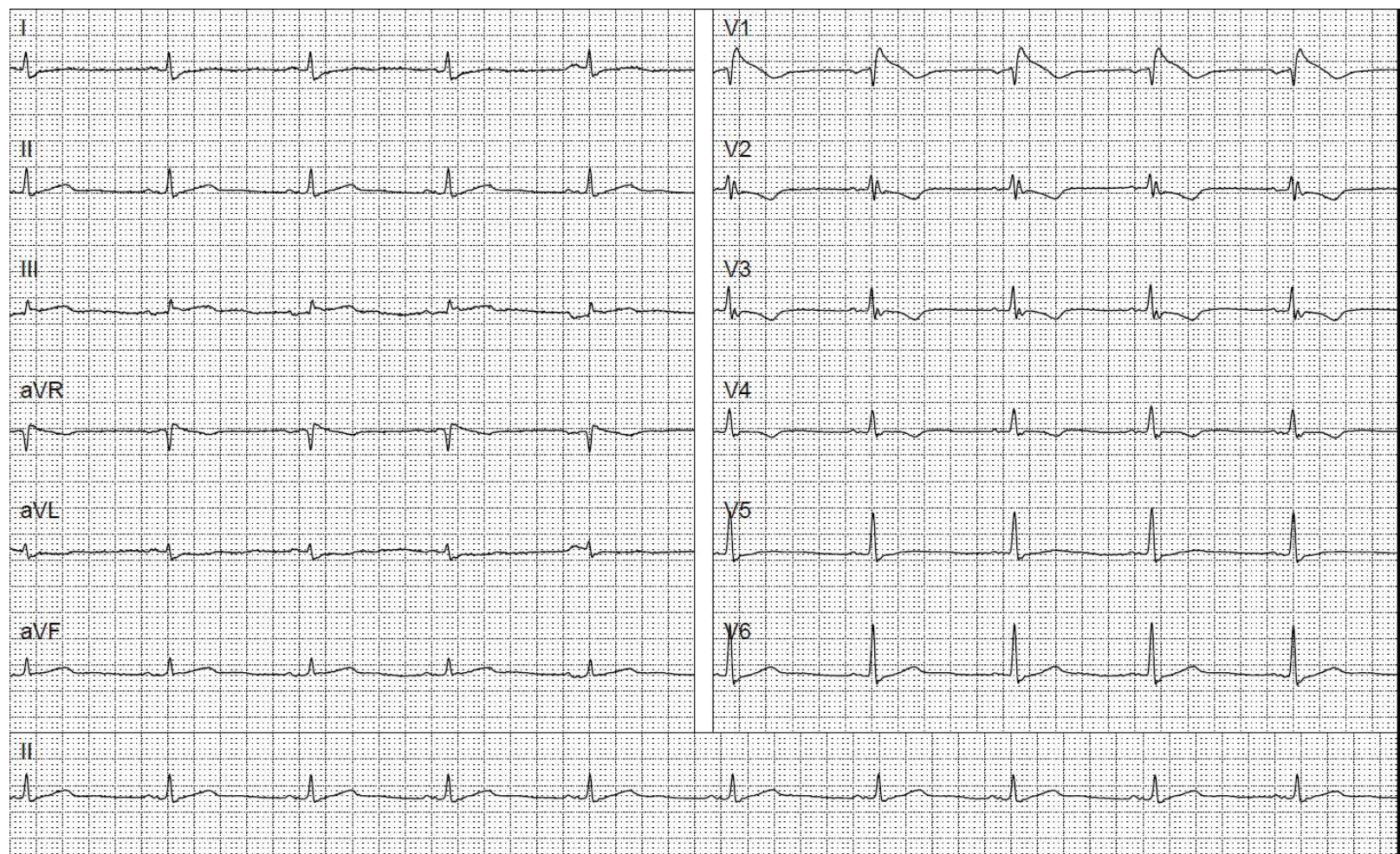

Figure 2: Standard resting 12-lead ECG at the current presentation. The precordial lead V1 shows an elevated J-point of $0.25 \mathrm{mV}$ with an elevated coved ST segment a negative T-wave. T-wave inversions are visible in V2 to V4. These findings are consistent with a type 1 Brugada ECG.

electrocardiographic criteria of Brugada ECG pattern [6], this rSr' finding cannot be interpreted as early stage of a Brugada pattern due to the peaked positive terminal $r$ ' and the identical QRS duration in V1 and V6.

The ECG performed during the current hospitalization fulfilled all criteria of a type 1 ECG pattern, showing a typical ST elevation of $2.5 \mathrm{~mm}$ and a coved ST segment with T-wave inversion in V1. The Corrado index [3], distinguishing between Brugada pattern and other pathologies with ST-elevations mimicking a Brugada pattern, was also positive.
Following to the guidelines of the European Society of Cardiology for the management of patients with ventricular arrhythmias and the prevention of sudden cardiac death [4], and the consensus statement on the diagnosis and management of patients with inherited primary arrhythmia syndromes [7], the ECG alone is sufficient for the diagnosis of a Brugada syndrome. However, in our patient, we have to interpret the ECG findings as a Brugada phenocopy [8]. Anselm, et al. published criteria to identify Brugada phenocopies, which include reversibility of the pattern after removal or disappearance of the provoking factor. There are case reports of extra-cardiac tumor compressing the RVOT. With removal of the tumor, the Brugada 


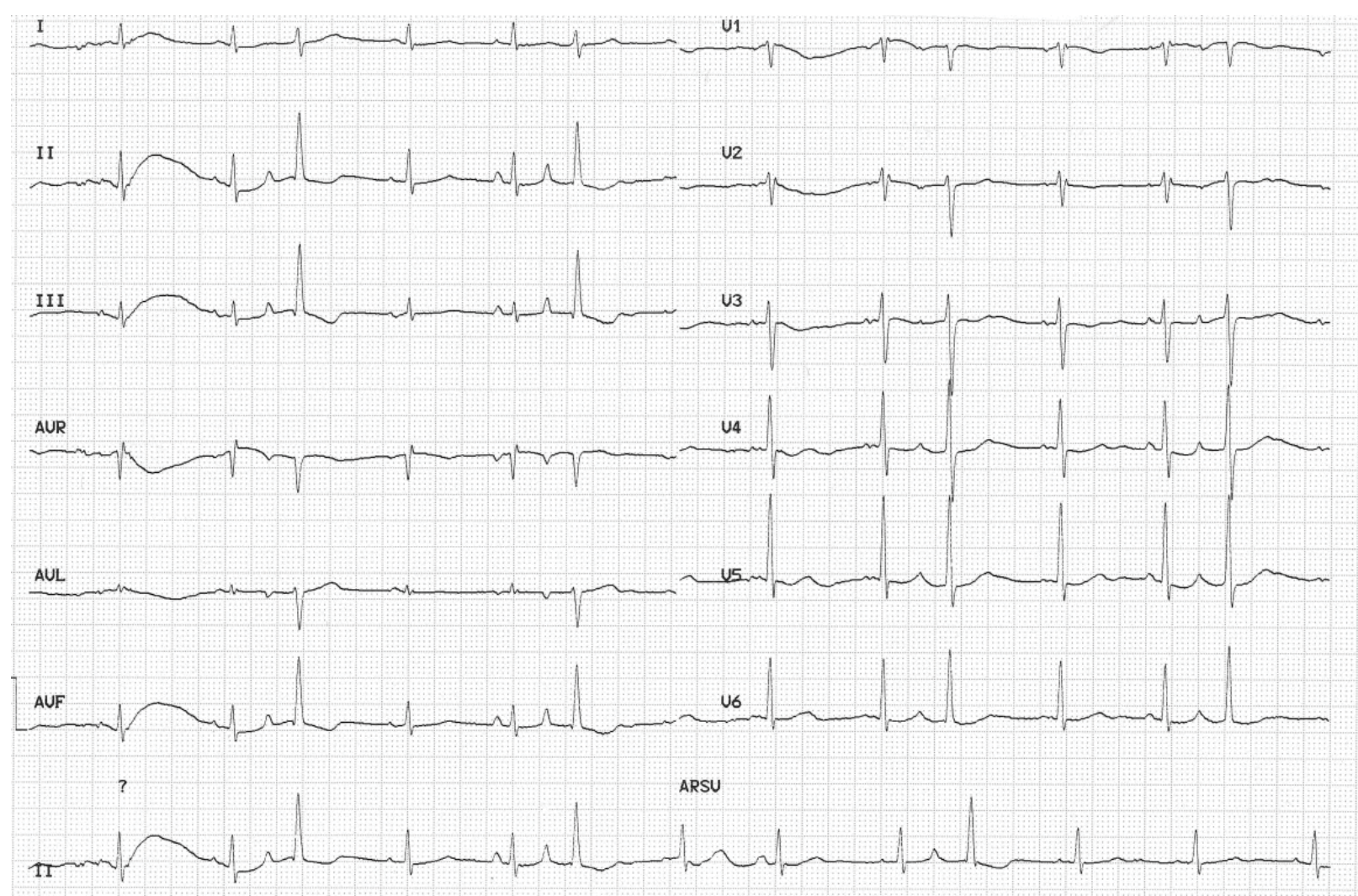

Figure 3: Standard resting 12-lead ECG 6 months before the current presentation without Brugada pattern.

pattern disappeared $[9,10]$. In our patient, a proof of Brugada phenocopy is missing because reversibility could not be shown. Thus a diagnosis of a Brugada phenocopy cannot be achieved based on these criteria [8]. However, the evolution of the ECG changes over time, the patient's history, and the localization of the mass in the right ventricular wall are highly suggestive for a Brugada phenocopy.

Patients with a Brugada phenocopy due to RVOT compression were described previously [10]. However, to our knowledge, this is the first reported case of a probable Brugada phenocopy secondary to a cardiac metastasis.

The pathophysiological evolution of the Brugada pattern by compression of the RVOT remains poorly understood. Alterations in the transmural repolarization gradient by the metastasis may contribute to this pattern [11].

The limitation of this case report is that it was not possible to proof a Brugada phenocopy based on published criteria. This is a well-known "diagnostic dilemma" [12]. However, considering the evolution of ECG in combination with the finding of an RVOT compression due to a cardiac metastasis, and the missing personal and family history for a Brugada syndrome strongly supports the hypothesis of a Brugada phenocopy.

\section{Financial Support}

The authors did not receive financial support for this work.

\section{References}

1. Bussani R, De-Giorgio F, Abbate A, et al. (2007) Cardiac metastases. J Clin Pathol 60: 27-34.

2. Cates CU, Virmani R, Vaughn WK, et al. (1986) Electrocardiographic markers of cardiac metastasis. Am Heart J 112: 1297-1303.

3. Corrado D, Pelliccia A, Heidbuchel H, et al. (2010) Recommendations for interpretation of 12-lead electrocardiogram in the athlete. Eur Heart $\mathrm{J} 31$ :
243-259.

4. Silvia G. Priori, Carina Blomström-Lundqvist, Andrea Mazzanti, et al. (2015) ESC Guidelines for the management of patients with ventricular arrhythmias and the prevention of sudden cardiac death. Eur Heart J 36: 2793-867.

5. Surawicz B, Childers R, Deal BJ, et al. (2009) AHA/ACCF/HRS Recommendations for the Standardization and Interpretation of the Electrocardiogram: Part III: Intraventricular Conduction Disturbances A Scientific StatementFrom the American Heart Association Electrocardiography and Arrhythmias Committee, Council on Clinical Cardiology; the American College of Cardiology Foundation; and the Heart Rhythm Society Endorsed by the International Society for Computerized Electrocardiology. J Am Coll Cardiol 53: 976-981.

6. Bayés de Luna A, Brugada J, Baranchuk A, et al. (2012) Current electrocardiographic criteria for diagnosis of Brugada pattern: a consensus report. J Electrocardiol 45: 433-442.

7. Priori SG, Wilde AA, Horie M, et al. (2013) HRS/EHRA/APHRS expert consensus statement on the diagnosis and management of patients with inherited primary arrhythmia syndromes: document endorsed by HRS EHRA, and APHRS in May 2013 and by ACCF, AHA, PACES, and AEPC in June 2013. Heart Rhythm 10: 1932-1963.

8. Anselm DD, Baranchuk A (2013) Brugada Phenocopy: redefinition and updated Classification. Am J Cardiol 111: 453

9. Yuji Nakazato, Takayasu Ohmura, Issei Shimada, et al. (2003) Brugada-like precordial ST elevation on ECG by anterior mediastinal infective mass lesion. Indian Pacing Electrophysiol J 3: 184

10. Asteriou C, Lazopoulos A, Giannoulis N, et al. (2013) Brugada-like ECG pattern due to giant mediastinal lipoma. Hippokratia 17: 368-369.

11. Arteyeva NV, Azarov JE (2016) The Role of Transmural Repolarization Gradient in the Inversion of Cardiac Electric Field: Model Study of ECG in Hypothermia. Ann. Noninvasive Electrocardiol.

12. Anselm DD, Gottschalk BH, Baranchuk A (2014) Brugada Phenocopies: Consideration of Morphologic Criteria and Early Findings From an International Registry. Can J Cardiol 30: 1511-1515. 